\title{
Predictive Models for Fast and Effective Profiling of Kinase Inhibitors
}

\author{
Alina Bora, ${ }^{1,2, \#}$ Sorin Avram, ${ }^{2, \#, *}$ Ionel Ciucanu, ${ }^{1}$ Marius Raica ${ }^{3}$, Stefana Avram ${ }^{4}$ \\ ${ }^{1}$ Department of Chemistry, West University of Timisoara, Faculty of Chemistry-Biology-Geography, 16 Pestalozzi Str., 300115, \\ Timisoara, Romania \\ ${ }^{2}$ Department of Computational Chemistry, Institute of Chemistry Timisoara of Romanian Academy, 24 Mihai Viteazu Avenue, \\ Timisoara, 300223, Romania
}

${ }^{3}$ Department of Microscopic Morphology/Histology, Angiogenesis Research Center, University of Medicine and Pharmacy "Victor Babes" Timisoara, 2 Eftimie Murgu, Timisoara, 300041, Romania

${ }^{4}$ Department Pharmacy II, Discip line of Pharmacognosy, University of Medicine and Pharmacy "Victor Babes” Timisoara, Faculty of Pharmacy, 2 Eftimie Murgu, Timisoara, 300041, Romania

\# These authors contributed equally to the article.

*Corresponding author. E-mail: sorin avram@acad-icht.tm.edu.ro or 5orin.4vram@gmail.com, Phone: +40-723-652-409.

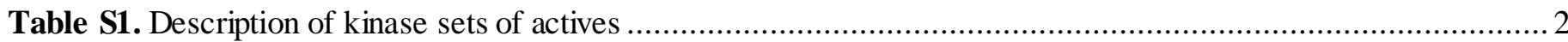

Table S2. Standardization steps and parameters applied to process molecular structures using module Chemaxon

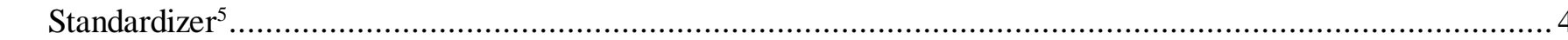

Table S3. Control parameters for random forest model generation using cForest ${ }^{6-9}$ in package "party" available in $\mathrm{R}$ statistical software system ${ }^{10}$.

Table S4. Number of active and inactive compounds (according to the corresponding data source) in the training and test sets

Table S5. Median, average and standard deviation of the number of compounds and the actives (computed per kinase set) ranked before the top $0.5 \% \mathrm{FP}$ in the external VS test sets

Table S6. Wilcoxon right shift $\mathrm{p}$-values $(\alpha=0.05)$ : row elements contain significantly greater values compared to column elements; median values are reported in parentheses ..........................................................

Table S7. Classification results of the kinase model prediction for kinase sets with actives and inactives defined as $\mathrm{pK}_{\mathrm{i}}$ $\geq 7$ and $\mathrm{pK}_{\mathrm{i}}<5$, respectively, in Metz et a $\mathrm{l}^{11}$ profiling results

Table S8. Classification results of the kinase model prediction for kinase sets with actives and inactives defined as scaled Activity $\% \leq 0.1$ and $>0.9$, respectively, in Anastassiadis et $\mathrm{l}^{12}$ profiling kinase sets

Figure S1. Density distribution plots of the activity values $\left(\mathrm{IC}_{50}\right.$ in $\left.\mu \mathrm{M}\right)$ and hot spot cutoff (red line) for the first three kinases (according to the ID number assigned in the paper) in cluster k1 (a), k2 (b), k3 (c) and k4 (d). The plot title contains the UniProt ID of the kinase and the ID number (in parenthesis) used in the paper.

Figure S2. Dendrograms resulted from Ward hierarchical clustering of 107 kinase sets in a first run (left), resulting in k4 (magenta) and a larger cluster of 103 kinase sets. These were clustered separately into 3 more groups (right) k2 (blue), k3 (red) and k1 (green).

Figure S3. Accuracy (Acc), sensitivity (Se) and specificity $(\mathrm{Sp})$ values of the internal (training sets) evaluation of the 107 kinase models.

Figure S4. Phylogenetic tree of the human kinases (generated using Kinome Render ${ }^{13,14}$ ) Target IDs and abbreviations of the kinases modeled in the current study are colored in red; two Atypical:PI3/PI4-kinase kinases, i.e., PIK3CA and PIK3CG could not be mapped and are not represented. Illustration reproduced courtesy of Cell Signaling Technology, Inc. (www.cellsignal.com).

Figure S5. The distributions of (the kinase-set values) eROCE (a, performed on the VS test sets) and AUC (b, performed on the classification test sets) according to the nine kinase groups.

Figure S6. Venn plot (created using the online software from Microarray Center CRP-Sante) ${ }^{15}$ of the chemical scaffolds (BMFs) contained in the PubChemKinIna (only inactives), ChEMBL (only kinase inhibitors) and ColBioS-FlavRC ${ }^{16}$ datasets. 
Table S1. Description of kinase sets of actives

\begin{tabular}{|c|c|c|c|c|c|c|c|c|c|c|}
\hline $\mathrm{ID}^{1}$ & $\begin{array}{l}\text { Uniprot } \\
\text { ID }^{2}\end{array}$ & GENE Name & $\begin{array}{l}\text { Kinase } \\
\text { group }\end{array}$ & $\begin{array}{l}\text { Kinase } \\
\text { Cluster }\end{array}$ & $\begin{array}{l}\text { Num of } \\
\text { Actives }\end{array}$ & $\begin{array}{l}\text { Num of } \\
\text { BMFs }^{3}\end{array}$ & $\begin{array}{l}\text { Actives } \\
\text { BMMF }^{4}\end{array}$ & $\begin{array}{l}\text { avg } \\
\text { TanD }\end{array}$ & $\begin{array}{l}\text { avg } \\
\mathrm{IC}_{50}{ }^{6}\end{array}$ & $\begin{array}{l}\text { Hotspot/ } \\
\text { IC }_{50} \\
\text { cutoff }\end{array}$ \\
\hline 1 & P35968 & KDR & TK & 1 & 2822 & 743 & 3.8 & 0.43 & 0.04 & $0.57 / 0.57$ \\
\hline 2 & P00533 & EGFR & $\mathrm{TK}$ & 1 & 2270 & 549 & 4.13 & 0.47 & 0.07 & $1.23 / 1.23$ \\
\hline 3 & Q16539 & MAPK14 & CMGC & 1 & 2206 & 494 & 4.47 & 0.43 & 0.04 & $0.48 / 0.48$ \\
\hline 4 & P42336 & PIK3CA & Atypical ${ }^{8}$ & 1 & 1391 & 381 & 3.65 & 0.47 & 0.11 & $1.36 / 1.36$ \\
\hline 5 & P12931 & SRC & $\mathrm{TK}$ & 1 & 1342 & 422 & 3.18 & 0.47 & 0.14 & $1.53 / 1.53$ \\
\hline 6 & P08581 & MET & TK & 1 & 1082 & 295 & 3.67 & 0.48 & 0.04 & $0.41 / 0.4$ \\
\hline 7 & P49841 & GSK3B & CMGC & 1 & 1031 & 273 & 3.78 & 0.43 & 0.11 & $1.27 / 1.27$ \\
\hline 8 & O14757 & CHEK1 & CAMK & 1 & 937 & 275 & 3.41 & 0.44 & 0.01 & $0.16 / 0.15$ \\
\hline 9 & O14965 & AURKA & Other & 1 & 880 & 339 & 2.6 & 0.45 & 0.07 & $0.9 / 0.89$ \\
\hline 10 & P04626 & ERBB2 & $\mathrm{TK}$ & 1 & 875 & 248 & 3.53 & 0.52 & 0.06 & $0.66 / 0.63$ \\
\hline 11 & P06239 & LCK & TK & 1 & 862 & 358 & 2.41 & 0.47 & 0.03 & $0.56 / 0.55$ \\
\hline 12 & P31749 & AKT1 & AGC & 1 & 840 & 224 & 3.75 & 0.47 & 0.06 & $0.74 / 0.74$ \\
\hline 13 & P48736 & PIK3CG & Atypical ${ }^{8}$ & 1 & 755 & 204 & 3.7 & 0.46 & 0.16 & $1.63 / 1.63$ \\
\hline 14 & P42345 & MTOR & Atypical $^{8}$ & 1 & 696 & 214 & 3.25 & 0.53 & 0.01 & $0.23 / 0.23$ \\
\hline 15 & P24941 & CDK2 & CMGC & 1 & 688 & 225 & 3.06 & 0.42 & 0.05 & $0.71 / 0.71$ \\
\hline 16 & P08069 & IGF1R & TK & 1 & 626 & 196 & 3.19 & 0.51 & 0.1 & $1.2 / 1.14$ \\
\hline 17 & P45983 & MAPK8 & CMGC & 1 & 596 & 156 & 3.82 & 0.43 & 0.11 & $1.46 / 1.45$ \\
\hline 18 & P06493 & CDK1 & CMGC & 1 & 578 & 190 & 3.04 & 0.44 & 0.16 & $1.52 / 1.5$ \\
\hline 19 & O14920 & IKBKB & Other & 1 & 576 & 156 & 3.69 & 0.47 & 0.1 & $1.39 / 1.34$ \\
\hline 20 & O60674 & JAK2 & $\mathrm{TK}$ & 1 & 570 & 199 & 2.86 & 0.45 & 0.05 & $0.45 / 0.44$ \\
\hline 21 & Q96GD4 & AURKB & Other & 1 & 559 & 225 & 2.48 & 0.47 & 0.05 & $0.49 / 0.49$ \\
\hline 22 & P36888 & FLT3 & $\mathrm{TK}$ & 1 & 536 & 217 & 2.47 & 0.44 & 0.03 & $0.31 / 0.31$ \\
\hline 23 & P17948 & FLT1 & TK & 1 & 504 & 159 & 3.17 & 0.43 & 0.24 & $1.59 / 1.59$ \\
\hline 24 & P09619 & PDGFRB & TK & 1 & 495 & 163 & 3.04 & 0.51 & 0.1 & $1.43 / 1.41$ \\
\hline 25 & Q02763 & TEK & TK & 1 & 449 & 119 & 3.77 & 0.5 & 0.1 & $1.16 / 1.13$ \\
\hline 26 & P10721 & KIT & $\mathrm{TK}$ & 1 & 443 & 152 & 2.91 & 0.44 & 0.03 & $0.31 / 0.3$ \\
\hline 27 & P53779 & MAPK10 & CMGC & 1 & 422 & 132 & 3.2 & 0.43 & 0.08 & $0.8 / 0.8$ \\
\hline 28 & P52333 & JAK3 & $\mathrm{TK}$ & 1 & 421 & 190 & 2.22 & 0.45 & 0.13 & $1.02 / 1$ \\
\hline 29 & P11362 & FGFR1 & $\mathrm{TK}$ & 1 & 418 & 127 & 3.29 & 0.48 & 0.08 & $1.04 / 1.03$ \\
\hline 30 & O75116 & ROCK2 & AGC & 1 & 416 & 124 & 3.35 & 0.42 & 0.02 & $0.28 / 0.27$ \\
\hline 31 & P00519 & ABL1 & $\mathrm{TK}$ & 1 & 395 & 160 & 2.47 & 0.48 & 0.07 & $1.39 / 1.36$ \\
\hline 32 & P11309 & PIM1 & CAMK & 1 & 387 & 135 & 2.87 & 0.43 & 0.03 & $0.47 / 0.47$ \\
\hline 33 & Q08881 & ITK & $\mathrm{TK}$ & 1 & 367 & 96 & 3.82 & 0.52 & 0.14 & $1.22 / 1.2$ \\
\hline 34 & P45984 & MAPK9 & CMGC & 1 & 363 & 115 & 3.16 & 0.44 & 0.14 & $1.21 / 1.2$ \\
\hline 35 & Q02750 & MAP2K1 & STE & 1 & 346 & 81 & 4.27 & 0.45 & 0.04 & $0.31 / 0.3$ \\
\hline 36 & P15056 & BRAF & TKL & 1 & 345 & 145 & 2.38 & 0.5 & 0.04 & $0.53 / 0.51$ \\
\hline 37 & P43405 & SYK & $\mathrm{TK}$ & 1 & 313 & 81 & 3.86 & 0.48 & 0.11 & $1.01 / 1$ \\
\hline 38 & P04049 & RAF1 & TKL & 1 & 295 & 84 & 3.51 & 0.45 & 0.07 & $0.7 / 0.7$ \\
\hline 39 & Q13464 & ROCK1 & AGC & 1 & 282 & 103 & 2.74 & 0.42 & 0.06 & $0.89 / 0.87$ \\
\hline 40 & Q9P1W9 & PIM2 & CAMK & 1 & 249 & 85 & 2.93 & 0.45 & 0.07 & $0.65 / 0.65$ \\
\hline 41 & O00311 & CDC7 & Other & 1 & 207 & 56 & 3.7 & 0.47 & 0.04 & $0.41 / 0.4$ \\
\hline 42 & P16234 & PDGFRA & $\mathrm{TK}$ & 1 & 159 & 53 & 3 & 0.45 & 0.03 & $0.34 / 0.34$ \\
\hline 43 & P04629 & NTRK1 & TK & 1 & 156 & 73 & 2.14 & 0.45 & 0.03 & $0.33 / 0.32$ \\
\hline 44 & Q02156 & PRKCE & AGC & 1 & 152 & 64 & 2.38 & 0.47 & 0.07 & $1.44 / 1.3$ \\
\hline
\end{tabular}




\begin{tabular}{|c|c|c|c|c|c|c|c|c|c|c|}
\hline 45 & P49840 & GSK3A & CMGC & 1 & 138 & 46 & 3 & 0.45 & 0.03 & $0.38 / 0.36$ \\
\hline 46 & P35916 & FLT4 & $\mathrm{TK}$ & 1 & 130 & 73 & 1.78 & 0.47 & 0.03 & $0.72 / 0.69$ \\
\hline 47 & Q5S007 & LRRK2 & TKL & 1 & 130 & 49 & 2.65 & 0.44 & 0.03 & $0.22 / 0.21$ \\
\hline 48 & Q86V86 & PIM3 & CAMK & 1 & 128 & 53 & 2.42 & 0.44 & 0.03 & $0.27 / 0.26$ \\
\hline 49 & P29597 & TYK2 & TK & 1 & 94 & 42 & 2.24 & 0.49 & 0.16 & $1.53 / 1.5$ \\
\hline 50 & P78527 & PRKDC & Atypical ${ }^{8}$ & 2 & 521 & 149 & 3.5 & 0.5 & 0.38 & $3.19 / 3.1$ \\
\hline 51 & P49137 & MAPKAPK2 & CAMK & 2 & 464 & 137 & 3.39 & 0.47 & 0.32 & $3.05 / 3$ \\
\hline 52 & P36897 & TGFBR1 & TKL & 2 & 397 & 69 & 5.75 & 0.56 & 0.03 & $0.33 / 0.32$ \\
\hline 53 & P06213 & INSR & TK & 2 & 344 & 134 & 2.57 & 0.49 & 0.38 & $2.9 / 2.9$ \\
\hline 54 & P54760 & EPHB4 & TK & 2 & 270 & 68 & 3.97 & 0.49 & 0.44 & $3.41 / 3.4$ \\
\hline 55 & P17252 & PRKCA & AGC & 2 & 251 & 102 & 2.46 & 0.49 & 0.4 & $3.39 / 3.3$ \\
\hline 56 & P11802 & CDK4 & CMGC & 2 & 250 & 86 & 2.91 & 0.44 & 0.26 & $2.46 / 2.3$ \\
\hline 57 & O15111 & CHUK & Other & 2 & 234 & 64 & 3.66 & 0.43 & 0.97 & $5.32 / 5.13$ \\
\hline 58 & Q05397 & PTK2 & $\mathrm{TK}$ & 2 & 203 & 70 & 2.9 & 0.49 & 0.45 & $3.59 / 3.5$ \\
\hline 59 & Q13627 & DYRK1A & CMGC & 2 & 193 & 55 & 3.51 & 0.47 & 0.28 & $2.53 / 2.5$ \\
\hline 60 & $\mathrm{P} 23458$ & JAK1 & $\mathrm{TK}$ & 2 & 175 & 84 & 2.08 & 0.45 & 0.18 & $2.06 / 2.06$ \\
\hline 61 & P07949 & RET & TK & 2 & 156 & 89 & 1.75 & 0.47 & 0.21 & $3.03 / 2.99$ \\
\hline 62 & P51812 & RPS6KA3 & AGC & 2 & 142 & 54 & 2.63 & 0.49 & 0.51 & $4.32 / 4.3$ \\
\hline 63 & P30530 & AXL & TK & 2 & 117 & 60 & 1.95 & 0.49 & 0.26 & $2.13 / 2$ \\
\hline 64 & P43403 & ZAP70 & TK & 2 & 112 & 47 & 2.38 & 0.53 & 0.34 & $3.48 / 3.3$ \\
\hline 65 & P49759 & CLK1 & CMGC & 2 & 110 & 28 & 3.93 & 0.48 & 0.22 & $2.6 / 2.5$ \\
\hline 66 & P48730 & CSNK1D & CK1 & 2 & 106 & 41 & 2.59 & 0.45 & 0.41 & $3.33 / 3.17$ \\
\hline 67 & Q15759 & MAPK11 & CMGC & 2 & 100 & 38 & 2.63 & 0.47 & 0.3 & $2.99 / 2.78$ \\
\hline 68 & Q9NWZ3 & IRAK4 & TKL & 2 & 97 & 43 & 2.26 & 0.51 & 0.46 & $3.34 / 3.03$ \\
\hline 69 & Q00535 & CDK5 & CMGC & 2 & 89 & 39 & 2.28 & 0.45 & 0.2 & $2.0 / 2.0$ \\
\hline 70 & P28482 & MAPK1 & CMGC & 2 & 85 & 36 & 2.36 & 0.55 & 0.28 & $3.21 / 3.11$ \\
\hline 71 & Q9HAZ1 & CLK4 & CMGC & 2 & 79 & 18 & 4.39 & 0.57 & 0.11 & $0.94 / 0.89$ \\
\hline 72 & Q13557 & CAMK2D & CAMK & 2 & 77 & 27 & 2.85 & 0.54 & 0.45 & $2.93 / 2.87$ \\
\hline 73 & P51955 & NEK2 & Other ${ }^{8}$ & 2 & 69 & 29 & 2.38 & 0.51 & 1.5 & $7.29 / 7.2$ \\
\hline 74 & Q13535 & ATR & Atypical ${ }^{9}$ & 2 & 66 & 16 & 4.13 & 0.6 & 0.52 & $3.51 / 3.26$ \\
\hline 75 & P06241 & FYN & $\mathrm{TK}$ & 2 & 59 & 42 & 1.4 & 0.48 & 0.4 & $2.87 / 2.8$ \\
\hline 76 & P50613 & CDK7 & CMGC & 2 & 51 & 22 & 2.32 & 0.5 & 0.41 & $2.37 / 2.3$ \\
\hline 77 & Q9UM73 & ALK & TK & 3 & 334 & 107 & 3.12 & 0.51 & 0.03 & $0.36 / 0.36$ \\
\hline 78 & Q05655 & PRKCD & AGC & 3 & 330 & 116 & 2.84 & 0.51 & 0.07 & $0.49 / 0.49$ \\
\hline 79 & P53350 & PLK1 & Other & 3 & 302 & 92 & 3.28 & 0.56 & 0.03 & $0.72 / 0.7$ \\
\hline 80 & Q04759 & PRKCQ & AGC & 3 & 297 & 99 & 3 & 0.54 & 0.01 & $0.13 / 0.13$ \\
\hline 81 & P31751 & AKT2 & AGC & 3 & 293 & 94 & 3.12 & 0.51 & 0.03 & $0.34 / 0.33$ \\
\hline 82 & P05771 & PRKCB & AGC & 3 & 199 & 73 & 2.73 & 0.55 & 0.04 & $0.77 / 0.77$ \\
\hline 83 & P08631 & $\mathrm{HCK}$ & TK & 3 & 193 & 63 & 3.06 & 0.62 & 0.11 & $1.23 / 1.22$ \\
\hline 84 & O96017 & CHEK2 & CAMK & 3 & 179 & 63 & 2.84 & 0.5 & 0.1 & $1.17 / 1.13$ \\
\hline 85 & O15530 & PDPK1 & AGC & 3 & 157 & 50 & 3.14 & 0.5 & 0.09 & $0.83 / 0.79$ \\
\hline 86 & P07948 & LYN & TK & 3 & 147 & 77 & 1.91 & 0.53 & 0.18 & $1.66 / 1.59$ \\
\hline 87 & Q06418 & TYRO3 & TK & 3 & 147 & 64 & 2.3 & 0.55 & 0.18 & $1.65 / 1.6$ \\
\hline 88 & P24723 & PRKCH & AGC & 3 & 130 & 43 & 3.02 & 0.51 & 0.02 & $0.25 / 0.25$ \\
\hline 89 & P41279 & MAP3K8 & STE & 3 & 123 & 34 & 3.62 & 0.59 & 0.08 & $0.9 / 0.83$ \\
\hline 90 & Q06187 & BTK & TK & 3 & 121 & 45 & 2.69 & 0.54 & 0.02 & $0.16 / 0.15$ \\
\hline 91 & Q9Y243 & AKT3 & AGC & 3 & 114 & 42 & 2.71 & 0.54 & 0.01 & $0.34 / 0.28$ \\
\hline 92 & P05129 & PRKCG & AGC & 3 & 106 & 44 & 2.41 & 0.57 & 0.27 & $2.05 / 2$ \\
\hline 93 & Q12866 & MERTK & $\mathrm{TK}$ & 3 & 97 & 44 & 2.2 & 0.58 & 0.02 & $0.26 / 0.23$ \\
\hline
\end{tabular}




\begin{tabular}{rlllrrrrrrr}
\hline 94 & P33981 & TTK & Other & 3 & 95 & 38 & 2.5 & 0.54 & 0.03 & $0.36 / 0.36$ \\
95 & Q9Y463 & DYRK1B & CMGC & 3 & 83 & 24 & 3.46 & 0.52 & 0.05 & $0.57 / 0.56$ \\
96 & Q9UHD2 & TBK1 & Other & 3 & 82 & 44 & 1.86 & 0.56 & 0.03 & $0.33 / 0.29$ \\
97 & Q13882 & PTK6 & TK & 3 & 72 & 30 & 2.4 & 0.6 & 0.01 & $0.15 / 0.12$ \\
98 & Q15139 & PRKD1 & CAMK & 3 & 71 & 32 & 2.22 & 0.58 & 0.04 & $0.53 / 0.53$ \\
99 & Q07912 & TNK2 & TK & 3 & 62 & 26 & 2.38 & 0.59 & 0.1 & $0.72 / 0.65$ \\
100 & Q9H4B4 & PLK3 & Other & 3 & 61 & 20 & 3.05 & 0.62 & 0.04 & $0.43 / 0.35$ \\
101 & Q9NYY3 & PLK2 & Other & 3 & 58 & 29 & 2 & 0.65 & 0.18 & $1.94 / 1.9$ \\
102 & Q14289 & PTK2B & TK & 3 & 56 & 29 & 1.93 & 0.55 & 0.06 & $0.55 / 0.43$ \\
103 & Q14164 & IKBKE & Other & 3 & 54 & 22 & 2.45 & 0.6 & 0.23 & $2.04 / 1.75$ \\
104 & P30291 & WEE1 & Other & 4 & 200 & 26 & 7.69 & 0.73 & 0.06 & $0.41 / 0.41$ \\
105 & Q05513 & PRKCZ & AGC & 4 & 55 & 22 & 2.5 & 0.46 & 3.1 & $10 / 9.6$ \\
106 & P34947 & GRK5 & AGC & 4 & 51 & 15 & 3.4 & 0.85 & 1.78 & $8.16 / 7.9$ \\
107 & O43318 & MAP3K7 & STE & 4 & 50 & 9 & 5.56 & 0.82 & 0.67 & $4.74 / 4.5$ \\
\hline
\end{tabular}

${ }^{1}$ identifiers used for kinases throughout the paper; ${ }^{2}$ UniProt $^{1,2}$ identifiers; ${ }^{3}$ number of Bemis-Murko Frameworks $(\mathrm{BMFs})^{3} ;{ }^{4}$ number of actives per BMF; ${ }^{5}$ average of Tanimoto distances per kinase data set; ${ }^{6}$ average of $\mathrm{IC}_{50}$ values per kinase data set; ${ }^{7}$ hotspot value and the real maximum $\mathrm{IC}_{50}$ value (for actives) per kinase set; ${ }^{8}$ although $\mathrm{NEK} 2$ is classified by UniProt ${ }^{1,2}$ in the NEK group ("Human and mouse protein kinases: classification and index"; version 2015_02; see in text), here, we considered it as a member of "Other" according to Manning et $a l^{4} ;^{8}$ Atypical:PI3/PI4kinase.

Table S2. Standardization steps and parameters applied to process molecular structures using module Chemaxon Standardizer ${ }^{5}$

\begin{tabular}{|c|c|c|}
\hline Step & Description & Parameter \\
\hline 1 & Remove Fragment & $\begin{array}{l}\text { Remove Fragment, Measure="atomCount", } \\
\text { Method="keepLargest" }\end{array}$ \\
\hline 2 & StripSalts & \\
\hline 3 & Dearomatize & \\
\hline 4 & Neutralize & \\
\hline 5 & Transformation & $\begin{array}{l}\text { Transform Isocyanate, Transform Nitroso, } \\
\text { Transform Phosphonic,Transform } \\
\text { Phosphonium Ylide, Transform Selenite, } \\
\text { Transform Silicate, Transform Sulfine, } \\
\text { Transform Sulfon, Transform Sulfonium Ylide, } \\
\text { Type="basic" }\end{array}$ \\
\hline
\end{tabular}

Table S3. Control parameters for random forest model generation using cForest, ${ }^{6-9}$ in package "party" available in R statistical software system ${ }^{10}$

\begin{tabular}{ll}
\hline Command & cforest_control(teststat $=$ "max", testtype = "Teststatistic", mincriterion = \\
& qnorm $(0.9)$, ntrees $=500$, mtry $=15)$
\end{tabular}


Table S4. Number of active and inactive compounds (according to the corresponding data source) in the training and test sets

\begin{tabular}{|c|c|c|c|c|c|c|c|c|c|}
\hline \multirow{2}{*}{$\mathrm{ID}^{1}$} & \multicolumn{4}{|c|}{ Training sets } & \multicolumn{4}{|c|}{ Classification test sets } & \multirow{2}{*}{$\begin{array}{l}\begin{array}{l}\text { Virtual } \\
\text { screening } \\
\text { test sets }\end{array} \\
\text { PubChem } \\
\text { KinIna }^{4} \\
\end{array}$} \\
\hline & $\begin{array}{c}\text { ChEMBL } \\
\text { Actives }\end{array}$ & $\begin{array}{c}\text { Total } \\
\text { Inactives }^{2}\end{array}$ & $\begin{array}{l}\text { PubChem } \\
\text { KinIna }\end{array}$ & $\begin{array}{l}\text { ChEMBL } \\
\text { inactives }\end{array}$ & $\begin{array}{l}\text { ChEMBL } \\
\text { Actives }\end{array}$ & $\begin{array}{c}\text { Total } \\
\text { Inactives }^{3}\end{array}$ & $\begin{array}{l}\text { PubChem } \\
\text { KinIna }\end{array}$ & $\begin{array}{l}\text { ChEMBL } \\
\text { Inactives }\end{array}$ & \\
\hline 1 & 2258 & 2258 & 1917 & 341 & 564 & 564 & 493 & 71 & 36547 \\
\hline 2 & 1816 & 1816 & 1331 & 485 & 454 & 454 & 324 & 130 & 37302 \\
\hline 3 & 1765 & 1765 & 1603 & 162 & 441 & 441 & 402 & 39 & 36952 \\
\hline 4 & 1113 & 1113 & 1008 & 105 & 278 & 278 & 253 & 25 & 37696 \\
\hline 5 & 1074 & 1074 & 813 & 261 & 268 & 268 & 202 & 66 & 37942 \\
\hline 6 & 866 & 866 & 813 & 53 & 216 & 216 & 205 & 11 & 37940 \\
\hline 7 & 825 & 825 & 712 & 113 & 206 & 206 & 179 & 27 & 38066 \\
\hline 8 & 750 & 750 & 673 & 77 & 187 & 187 & 169 & 18 & 38115 \\
\hline 9 & 704 & 704 & 626 & 78 & 176 & 176 & 162 & 14 & 38169 \\
\hline 10 & 700 & 700 & 642 & 58 & 175 & 175 & 158 & 17 & 38157 \\
\hline 11 & 690 & 690 & 598 & 92 & 172 & 172 & 144 & 28 & 38215 \\
\hline 12 & 672 & 672 & 544 & 128 & 168 & 168 & 142 & 26 & 38271 \\
\hline 13 & 604 & 604 & 547 & 57 & 151 & 151 & 128 & 23 & 38282 \\
\hline 14 & 557 & 557 & 513 & 44 & 139 & 139 & 129 & 10 & 38315 \\
\hline 15 & 550 & 550 & 455 & 95 & 138 & 137 & 107 & 30 & 38394 \\
\hline 16 & 501 & 501 & 439 & 62 & 125 & 125 & 112 & 13 & 38406 \\
\hline 17 & 477 & 477 & 435 & 42 & 119 & 119 & 111 & 8 & 38411 \\
\hline 18 & 462 & 462 & 338 & 124 & 116 & 115 & 81 & 34 & 38537 \\
\hline 19 & 461 & 461 & 387 & 74 & 115 & 115 & 94 & 21 & 38476 \\
\hline 20 & 456 & 456 & 432 & 24 & 114 & 114 & 103 & 11 & 38422 \\
\hline 21 & 447 & 447 & 392 & 55 & 112 & 111 & 106 & 5 & 38458 \\
\hline 22 & 429 & 429 & 411 & 18 & 107 & 107 & 101 & 6 & 38445 \\
\hline 23 & 403 & 403 & 347 & 56 & 101 & 100 & 86 & 14 & 38523 \\
\hline 24 & 396 & 396 & 305 & 91 & 99 & 99 & 70 & 29 & 38582 \\
\hline 25 & 359 & 359 & 314 & 45 & 90 & 89 & 76 & 13 & 38566 \\
\hline 26 & 354 & 354 & 340 & 14 & 89 & 88 & 84 & 4 & 38532 \\
\hline 27 & 338 & 338 & 289 & 49 & 84 & 84 & 74 & 10 & 38594 \\
\hline 28 & 337 & 337 & 285 & 52 & 84 & 84 & 72 & 12 & 38600 \\
\hline 29 & 334 & 334 & 271 & 63 & 84 & 83 & 67 & 16 & 38618 \\
\hline 30 & 333 & 333 & 313 & 20 & 83 & 83 & 78 & 5 & 38566 \\
\hline 31 & 316 & 316 & 238 & 78 & 79 & 79 & 66 & 13 & 38653 \\
\hline 32 & 310 & 310 & 302 & 8 & 77 & 77 & 75 & 2 & 38580 \\
\hline 33 & 294 & 294 & 246 & 48 & 73 & 73 & 52 & 21 & 38659 \\
\hline 34 & 290 & 290 & 266 & 24 & 73 & 72 & 68 & 4 & 38622 \\
\hline 35 & 277 & 277 & 266 & 11 & 69 & 69 & 66 & 3 & 38625 \\
\hline 36 & 276 & 276 & 264 & 12 & 69 & 69 & 68 & 1 & 38625 \\
\hline 37 & 250 & 250 & 231 & 19 & 63 & 62 & 59 & 3 & 38666 \\
\hline 38 & 236 & 236 & 224 & 12 & 59 & 59 & 55 & 4 & 38678 \\
\hline 39 & 226 & 226 & 206 & 20 & 56 & 56 & 50 & 6 & 38701 \\
\hline 40 & 199 & 199 & 185 & 14 & 50 & 49 & 48 & 1 & 38723 \\
\hline 41 & 166 & 166 & 153 & 13 & 41 & 41 & 39 & 2 & 38765 \\
\hline 42 & 127 & 127 & 123 & 4 & 32 & 31 & 29 & 2 & 38804 \\
\hline
\end{tabular}




\begin{tabular}{|c|c|c|c|c|c|c|c|c|c|}
\hline 43 & 125 & 125 & 123 & 2 & 31 & 31 & 30 & 1 & 38804 \\
\hline 44 & 122 & 122 & 84 & 38 & 30 & 30 & 23 & 7 & 38850 \\
\hline 45 & 110 & 110 & 105 & 5 & 28 & 27 & 26 & 1 & 38825 \\
\hline 46 & 104 & 104 & 101 & 3 & 26 & 26 & 26 & 0 & 38830 \\
\hline 47 & 104 & 104 & 98 & 6 & 26 & 26 & 25 & 1 & 38834 \\
\hline 48 & 102 & 102 & 100 & 2 & 26 & 25 & 24 & 1 & 38832 \\
\hline 49 & 75 & 75 & 70 & 5 & 19 & 18 & 18 & 0 & 38868 \\
\hline 50 & 417 & 417 & 389 & 28 & 104 & 104 & 96 & 8 & 38472 \\
\hline 51 & 371 & 371 & 267 & 104 & 93 & 92 & 68 & 24 & 38621 \\
\hline 52 & 318 & 318 & 303 & 15 & 79 & 79 & 79 & 0 & 38575 \\
\hline 53 & 275 & 275 & 226 & 49 & 69 & 68 & 52 & 16 & 38678 \\
\hline 54 & 216 & 216 & 157 & 59 & 54 & 54 & 39 & 15 & 38761 \\
\hline 55 & 201 & 201 & 146 & 55 & 50 & 50 & 32 & 18 & 38779 \\
\hline 56 & 200 & 200 & 173 & 27 & 50 & 50 & 39 & 11 & 38745 \\
\hline 57 & 187 & 187 & 154 & 33 & 47 & 46 & 32 & 14 & 38770 \\
\hline 58 & 162 & 162 & 130 & 32 & 41 & 40 & 33 & 7 & 38793 \\
\hline 59 & 154 & 154 & 135 & 19 & 39 & 38 & 35 & 3 & 38786 \\
\hline 60 & 140 & 140 & 134 & 6 & 35 & 35 & 35 & 0 & 38788 \\
\hline 61 & 125 & 125 & 103 & 22 & 31 & 31 & 26 & 5 & 38828 \\
\hline 62 & 114 & 114 & 107 & 7 & 28 & 28 & 23 & 5 & 38827 \\
\hline 63 & 94 & 94 & 63 & 31 & 23 & 23 & 19 & 4 & 38875 \\
\hline 64 & 90 & 90 & 68 & 22 & 22 & 22 & 17 & 5 & 38872 \\
\hline 65 & 88 & 88 & 88 & 0 & 22 & 22 & 22 & 0 & 38847 \\
\hline 66 & 85 & 85 & 81 & 4 & 21 & 21 & 20 & 1 & 38856 \\
\hline 67 & 80 & 80 & 80 & 0 & 20 & 20 & 19 & 1 & 38858 \\
\hline 68 & 78 & 78 & 70 & 8 & 19 & 19 & 16 & 3 & 38871 \\
\hline 69 & 71 & 71 & 65 & 6 & 18 & 17 & 14 & 3 & 38877 \\
\hline 70 & 68 & 68 & 59 & 9 & 17 & 17 & 17 & 0 & 38881 \\
\hline 71 & 63 & 63 & 54 & 9 & 16 & 15 & 10 & 5 & 38892 \\
\hline 72 & 62 & 62 & 56 & 6 & 15 & 15 & 10 & 5 & 38891 \\
\hline 73 & 55 & 55 & 39 & 16 & 14 & 13 & 12 & 1 & 38905 \\
\hline 74 & 53 & 53 & 42 & 11 & 13 & 13 & 13 & 0 & 38902 \\
\hline 75 & 47 & 47 & 33 & 14 & 12 & 11 & 8 & 3 & 38915 \\
\hline 76 & 41 & 41 & 37 & 4 & 10 & 10 & 10 & 0 & 38910 \\
\hline 77 & 267 & 267 & 255 & 12 & 67 & 66 & 66 & 0 & 38635 \\
\hline 78 & 264 & 264 & 249 & 15 & 66 & 66 & 63 & 3 & 38645 \\
\hline 79 & 242 & 242 & 214 & 28 & 60 & 60 & 51 & 9 & 38692 \\
\hline 80 & 238 & 238 & 233 & 5 & 59 & 59 & 58 & 1 & 38666 \\
\hline 81 & 234 & 234 & 200 & 34 & 59 & 58 & 51 & 7 & 38705 \\
\hline 82 & 159 & 159 & 132 & 27 & 40 & 39 & 32 & 7 & 38792 \\
\hline 83 & 154 & 154 & 129 & 25 & 39 & 38 & 29 & 9 & 38798 \\
\hline 84 & 143 & 143 & 128 & 15 & 36 & 35 & 27 & 8 & 38801 \\
\hline 85 & 126 & 126 & 115 & 11 & 31 & 31 & 31 & 0 & 38811 \\
\hline 86 & 118 & 118 & 106 & 12 & 29 & 29 & 26 & 3 & 38825 \\
\hline 87 & 118 & 118 & 95 & 23 & 29 & 29 & 23 & 6 & 38839 \\
\hline 88 & 104 & 104 & 99 & 5 & 26 & 26 & 25 & 1 & 38833 \\
\hline 89 & 98 & 98 & 75 & 23 & 25 & 24 & 19 & 5 & 38862 \\
\hline 90 & 97 & 97 & 89 & 8 & 24 & 24 & 22 & 2 & 38846 \\
\hline 91 & 91 & 91 & 72 & 19 & 23 & 22 & 21 & 1 & 38863 \\
\hline 92 & 85 & 85 & 82 & 3 & 21 & 21 & 20 & 1 & 38855 \\
\hline 93 & 78 & 78 & 74 & 4 & 19 & 19 & 16 & 3 & 38867 \\
\hline
\end{tabular}




$\begin{array}{llllllllll}94 & 76 & 76 & 76 & 0 & 19 & 19 & 19 & 0 & 38862 \\ 95 & 66 & 66 & 66 & 0 & 17 & 16 & 16 & 0 & 38874 \\ 96 & 66 & 66 & 62 & 4 & 16 & 16 & 15 & 1 & 38880 \\ 97 & 58 & 58 & 55 & 3 & 14 & 14 & 10 & 4 & 38892 \\ 98 & 57 & 57 & 57 & 0 & 14 & 14 & 14 & 0 & 38886 \\ 99 & 50 & 50 & 50 & 0 & 12 & 12 & 12 & 0 & 38895 \\ 100 & 49 & 49 & 49 & 0 & 12 & 12 & 12 & 0 & 38896 \\ 101 & 46 & 46 & 46 & 0 & 12 & 11 & 11 & 0 & 38899 \\ 102 & 45 & 45 & 45 & 0 & 11 & 11 & 11 & 0 & 38901 \\ 103 & 43 & 43 & 34 & 9 & 11 & 10 & 9 & 1 & 38913 \\ 104 & 160 & 160 & 138 & 22 & 40 & 40 & 35 & 5 & 38784 \\ 105 & 44 & 44 & 21 & 23 & 11 & 11 & 4 & 7 & 38932 \\ 106 & 41 & 41 & 40 & 1 & 10 & 10 & 8 & 2 & 38909 \\ 107 & 40 & 40 & 39 & 1 & 10 & 10 & 10 & 0 & 38908\end{array}$

1 Kinase identification used throughout the article; ${ }^{2}$ Sums of inactives from PubChemKinIna and ChEMBL inactives used for training; ${ }^{3}$ inactives from PubChemKinIna and ChEMBL inactives used for classification testing; ${ }^{4}$ virtual screening test sets are composed from ChEMBL actives (used in the classification test sets) and PubChemKinIna set (after removing inactives used for modeling).

Table S5. Median, average and standard deviation of the number of compounds and the actives (computed per kinase set) ranked before the top $0.5 \% \mathrm{FP}$ in the external VS test sets

\begin{tabular}{cccc}
\hline Cluster & Num. of compounds & Num. of actives retrieved & Novel Actives $^{1}$ \\
\hline k1 & $278 / 302 / 97.9$ & $83 / 107 / 100.2$ & $16 / 19 / 17.5$ \\
k2 & $217 / 225 / 23.0$ & $20 / 28 / 23.6$ & $5 / 5 / 4.8$ \\
k3 & $219 / 224 / 16.8$ & $22 / 27 / 17.2$ & $4 / 5 / 3.8$ \\
k4 & $206 / 213 / 13.8$ & $9 / 17 / 14.34$ & $1 / 2 / 1.7$ \\
\hline All & $232 / 259 / 77.9$ & $36 / 64 / 79.9$ & $6 / 12 / 14.1$ \\
\hline
\end{tabular}

${ }^{1}$ Novel in the sense that these actives share BMFs which were not used in training

Table S6. Wilcoxon right shift $p$-values $(\alpha=0.05)$ : row elements contain significantly higher values compared to column elements; median values are reported in parentheses

\begin{tabular}{|c|c|c|c|c|c|}
\hline \multirow{5}{*}{ Internal Classification } & Acc & $\mathrm{k} 1$ & $\mathrm{k} 2$ & $\mathrm{k} 3$ & $\mathrm{k} 4$ \\
\hline & $\mathrm{k} 1(0.8725)$ & & $\mathbf{0}$ & 1 & 0.896 \\
\hline & $\mathrm{k} 2(0.8308)$ & 1 & & 1 & 0.923 \\
\hline & k3 (0.9167) & $\mathbf{0}$ & $\mathbf{0}$ & & 0.605 \\
\hline & $\mathrm{k} 4(0.8308)$ & 0.105 & 0.088 & 0.418 & \\
\hline \multirow{5}{*}{$\begin{array}{c}\text { External Classification test } \\
\text { set }\end{array}$} & Acc & $\mathrm{k} 1$ & $\mathrm{k} 2$ & k3 & $\mathrm{k} 4$ \\
\hline & $\mathrm{k} 1(0.8919)$ & & 0.025 & 0.991 & 0.518 \\
\hline & k2 (0.8676) & 0.975 & & 1 & 0.727 \\
\hline & k3 (0.9167) & 0.008 & 0.001 & & 0.185 \\
\hline & $\mathrm{k} 4(0.8919)$ & 0.489 & 0.284 & 0.823 & \\
\hline
\end{tabular}




\begin{tabular}{|c|c|c|c|c|c|}
\hline & $\mathrm{Sp}$ & \multirow[t]{2}{*}{$\mathrm{k} 1$} & \multirow{2}{*}{$\begin{array}{c}\mathrm{k} 2 \\
0.145\end{array}$} & \multirow{2}{*}{$\frac{\mathrm{k} 3}{0.811}$} & \multirow{2}{*}{$\begin{array}{c}\mathrm{k} 4 \\
0.336\end{array}$} \\
\hline & $\mathrm{k} 1(0.9200)$ & & & & \\
\hline & $\mathrm{k} 2(0.9038)$ & 0.852 & & 0.909 & 0.349 \\
\hline & k3 (0.9487) & 0.189 & 0.093 & & 0.222 \\
\hline & $\mathrm{k} 4(0.8386)$ & 0.662 & 0.67 & 0.784 & \\
\hline & & & & & \\
\hline & $\mathrm{Se}$ & k1 & k2 & $\mathrm{k} 3$ & $\mathrm{k} 4$ \\
\hline & $\mathrm{k} 1(0.8571)$ & & 0.136 & 1 & 0.933 \\
\hline & $\mathrm{k} 2(0.8421)$ & 0.868 & & 0.999 & 0.923 \\
\hline & k3 (0.9167) & $\mathbf{0}$ & 0.001 & & 0.534 \\
\hline & $\mathrm{k} 4(0.9125)$ & 0.071 & 0.078 & 0.479 & \\
\hline & AUC & $\mathrm{k} 1$ & k2 & k3 & $\mathrm{k} 4$ \\
\hline & k1 (0.960) & & 0.058 & 0.986 & 0.376 \\
\hline & $\mathrm{k} 2(0.946)$ & 0.937 & & 0.996 & 0.421 \\
\hline & k3 (0.969) & 0.017 & 0.005 & & 0.185 \\
\hline & $\mathrm{k} 4(0.950)$ & 0.638 & 0.601 & 0.824 & \\
\hline \multirow{11}{*}{$\begin{array}{l}\text { External Virtual Screening } \\
\text { test set }\end{array}$} & eROCE & $\mathrm{k} 1$ & $\mathrm{k} 2$ & k3 & $\mathrm{k} 4$ \\
\hline & $\mathrm{k} 1(0.9127)$ & & 0.101 & 1 & 0.92 \\
\hline & k2 (0.8900) & 0.903 & & 1 & 0.933 \\
\hline & k3 (0.9585) & $\mathbf{0}$ & $\mathbf{0}$ & & 0.495 \\
\hline & $\mathrm{k} 4(0.9639)$ & 0.083 & 0.079 & 0.511 & \\
\hline & & & & & \\
\hline & TPR at $0.5 \% \mathrm{FP}$ & $\mathrm{k} 1$ & $\mathrm{k} 2$ & $\mathrm{k} 3$ & $\mathrm{k} 4$ \\
\hline & $\mathrm{k} 1(0.8435)$ & & 0.028 & 1 & 0.959 \\
\hline & $\mathrm{k} 2(0.8000)$ & 0.971 & & 1 & 0.979 \\
\hline & k3 (0.9375) & $\mathbf{0}$ & $\mathbf{0}$ & & 0.366 \\
\hline & $\mathrm{k} 4(0.9250)$ & 0.042 & 0.022 & 0.647 & \\
\hline
\end{tabular}

Table S7. Classification results of the kinase model prediction for kinase sets with actives and inactives defined as $\mathrm{pK}_{\mathrm{i}} \geq 7$ and $\mathrm{pK}_{\mathrm{i}}<5$, respectively, in Metz et a ${ }^{11}$ profiling results

\begin{tabular}{lccccccc}
\hline Uniprot $\mathrm{ID}^{1}$ & $\mathrm{Se}$ & $\mathrm{Sp}$ & Acc & AUC & SD & Actives & Inactives \\
\hline P35968 & 0.922 & 0.836 & 0.896 & 0.941 & 0.209 & 154 & 67 \\
P00533 & 0.72 & 1 & 0.8 & 0.86 & 0.311 & 25 & 10 \\
P08581 & 0.743 & 1 & 0.889 & 0.858 & 0.294 & 35 & 46 \\
P49841 & 0.605 & 0.912 & 0.663 & 0.756 & 0.359 & 147 & 34 \\
O14757 & 0.514 & 1 & 0.791 & 0.807 & 0.335 & 37 & 49 \\
O14965 & 0.782 & 0.938 & 0.832 & 0.872 & 0.277 & 133 & 64 \\
P06239 & 0.847 & 0.917 & 0.856 & 0.924 & 0.209 & 85 & 12 \\
P31749 & 0.889 & 1 & 0.972 & 0.889 & 0.333 & 9 & 27 \\
P08069 & 0.667 & 1 & 0.812 & 0.811 & 0.316 & 27 & 21 \\
P45983 & 0.73 & 0.943 & 0.833 & 0.9 & 0.22 & 37 & 35 \\
\hline
\end{tabular}




\begin{tabular}{|c|c|c|c|c|c|c|c|}
\hline O60674 & 0.845 & 0.971 & 0.886 & 0.928 & 0.2 & 71 & 34 \\
\hline Q96GD4 & 0.573 & 0.933 & 0.625 & 0.748 & 0.36 & 178 & 30 \\
\hline P36888 & 0.757 & 1 & 0.788 & 0.937 & 0.192 & 189 & 28 \\
\hline P17948 & 0.783 & 1 & 0.811 & 0.916 & 0.223 & 175 & 26 \\
\hline P09619 & 0.75 & 0.792 & 0.774 & 0.818 & 0.325 & 40 & 53 \\
\hline P52333 & 0.894 & 0.957 & 0.914 & 0.934 & 0.171 & 47 & 23 \\
\hline P11362 & 0.73 & 0.981 & 0.836 & 0.912 & 0.244 & 74 & 54 \\
\hline P00519 & 0.771 & 0.943 & 0.829 & 0.932 & 0.165 & 70 & 35 \\
\hline P11309 & 0.514 & 0.941 & 0.761 & 0.726 & 0.356 & 37 & 51 \\
\hline Q08881 & 0.25 & 1 & 0.413 & 0.978 & 0.093 & 36 & 10 \\
\hline P45984 & 0.729 & 0.929 & 0.767 & 0.914 & 0.144 & 59 & 14 \\
\hline Q02750 & 0.316 & 1 & 0.745 & 0.944 & 0.109 & 19 & 32 \\
\hline Q13464 & 0.417 & 1 & 0.537 & 0.793 & 0.354 & 108 & 28 \\
\hline P16234 & 0.818 & 0.781 & 0.8 & 0.857 & 0.305 & 33 & 32 \\
\hline P04629 & 0.851 & 0.935 & 0.885 & 0.929 & 0.21 & 47 & 31 \\
\hline P49840 & 0.243 & 0.968 & 0.406 & 0.794 & 0.237 & 107 & 31 \\
\hline Q5S007 & 0.547 & 1 & 0.718 & 0.789 & 0.377 & 53 & 32 \\
\hline Q86V86 & 0.381 & 1 & 0.683 & 0.657 & 0.377 & 21 & 20 \\
\hline P29597 & 0.409 & 1 & 0.618 & 0.864 & 0.225 & 22 & 12 \\
\hline P06213 & 0.792 & 1 & 0.891 & 0.998 & 0.009 & 24 & 22 \\
\hline Q05397 & 0.633 & 1 & 0.817 & 0.927 & 0.144 & 30 & 30 \\
\hline Q13627 & 0.373 & 0.679 & 0.467 & 0.514 & 0.348 & 126 & 56 \\
\hline P07949 & 0.824 & 0.957 & 0.858 & 0.941 & 0.163 & 136 & 47 \\
\hline P51812 & 0.52 & 0.95 & 0.711 & 0.852 & 0.254 & 50 & 40 \\
\hline Q00535 & 0.526 & 0.84 & 0.59 & 0.757 & 0.305 & 97 & 25 \\
\hline Q9HAZ1 & 0.547 & 0.548 & 0.547 & 0.542 & 0.322 & 234 & 31 \\
\hline P51955 & 0.667 & 1 & 0.821 & 0.79 & 0.346 & 15 & 13 \\
\hline P06241 & 0.38 & 0 & 0.333 & 0.168 & 0.286 & 71 & 10 \\
\hline P50613 & 0.4 & 1 & 0.633 & 0.915 & 0.228 & 55 & 35 \\
\hline Q9UM73 & 0.884 & 0.974 & 0.926 & 0.945 & 0.177 & 43 & 38 \\
\hline Q04759 & 0.238 & 1 & 0.805 & 0.867 & 0.234 & 21 & 61 \\
\hline P31751 & 0.778 & 1 & 0.946 & 0.889 & 0.333 & 9 & 28 \\
\hline Q06418 & 0.091 & 1 & 0.701 & 0.861 & 0.28 & 22 & 45 \\
\hline Q06187 & 0.75 & 1 & 0.837 & 0.902 & 0.288 & 28 & 15 \\
\hline Q9Y243 & 0.667 & 1 & 0.897 & 0.923 & 0.267 & 12 & 27 \\
\hline Q9Y463 & 0.289 & 0.518 & 0.426 & 0.36 & 0.305 & 38 & 56 \\
\hline Q9UHD2 & 0.455 & 0.944 & 0.759 & 0.854 & 0.188 & 11 & 18 \\
\hline Q13882 & 0.2 & 1 & 0.586 & 0.752 & 0.351 & 15 & 14 \\
\hline Q16539 & 0.688 & 0.92 & 0.829 & 0.862 & 0.305 & 16 & 25 \\
\hline Q07912 & 0.318 & 1 & 0.688 & 0.832 & 0.344 & 22 & 26 \\
\hline Q9H4B4 & 0.318 & 1 & 0.681 & 0.902 & 0.241 & 22 & 25 \\
\hline Q14164 & 0.333 & 1 & 0.829 & 0.786 & 0.317 & 9 & 26 \\
\hline
\end{tabular}


Table S8. Classification results of the kinase model prediction for kinase sets with actives and inactives defined as scaled Activity $\% \leq 0.1$ and $>0.9$, respectively, in Anastassiadis et a ${ }^{12}$ profiling kinase sets

\begin{tabular}{lccccccc}
\hline Uniprot ID $^{1}$ & $\mathrm{Se}$ & $\mathrm{Sp}$ & Acc & AUC & SD & Actives & Inactives \\
\hline P00533 & 1 & 0.9 & 0.941 & 0.996 & 0.013 & 14 & 20 \\
P49841 & 0.833 & 0.667 & 0.75 & 0.820 & 0.221 & 12 & 12 \\
P24941cA & 0.461 & 0.9 & 0.652 & 0.885 & 0.121 & 13 & 10 \\
Q96GD4 & 0.214 & 0.884 & 0.771 & 0.553 & 0.312 & 14 & 69 \\
P36888 & 0.382 & 0.875 & 0.54 & 0.835 & 0.185 & 34 & 16 \\
P04629 & 0.75 & 0.96 & 0.892 & 0.937 & 0.086 & 12 & 25 \\
P07949 & 0.609 & 0.822 & 0.75 & 0.835 & 0.176 & 23 & 45 \\
Q00535p25 & 0.539 & 0.786 & 0.667 & 0.841 & 0.117 & 13 & 14 \\
Q00535p35 & 0.417 & 0.824 & 0.655 & 0.627 & 0.360 & 12 & 17 \\
Q07912 & 0.143 & 0.938 & 0.696 & 0.777 & 0.257 & 14 & 32 \\
\hline
\end{tabular}


a)

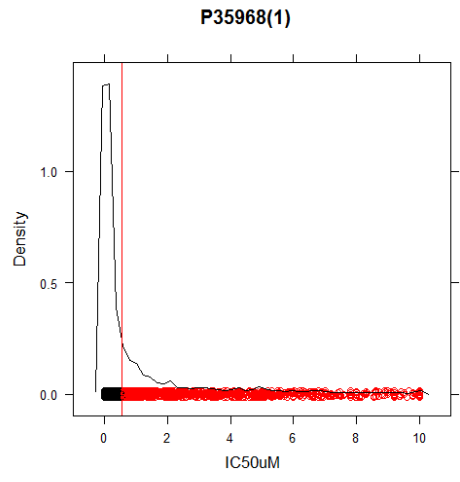

b)

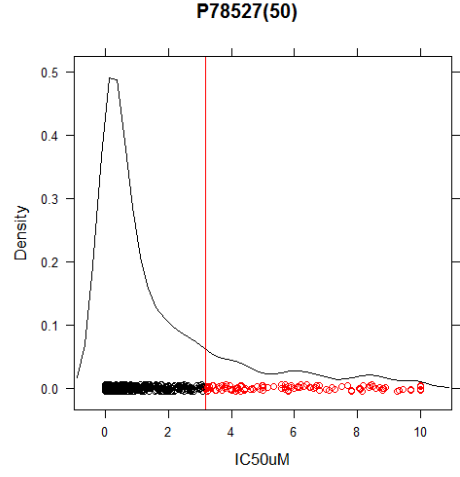

c)

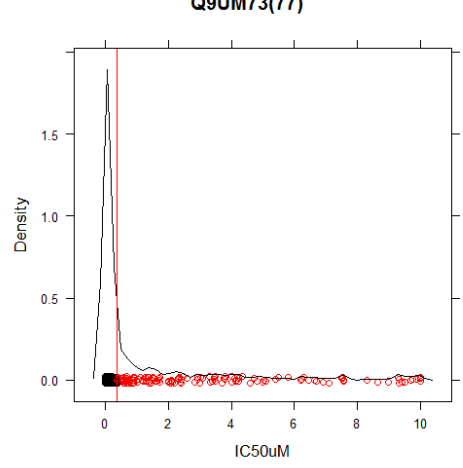

d)

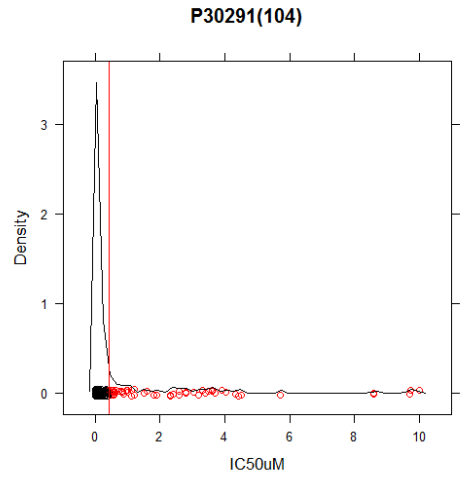

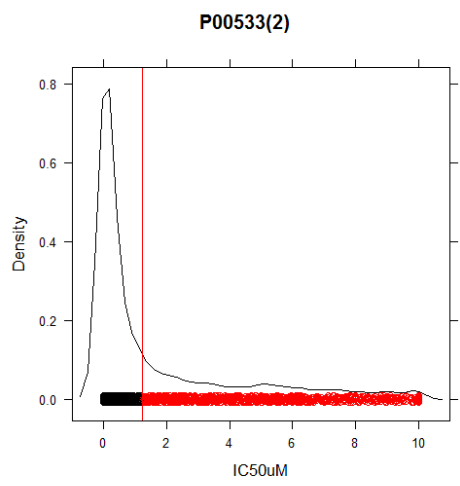
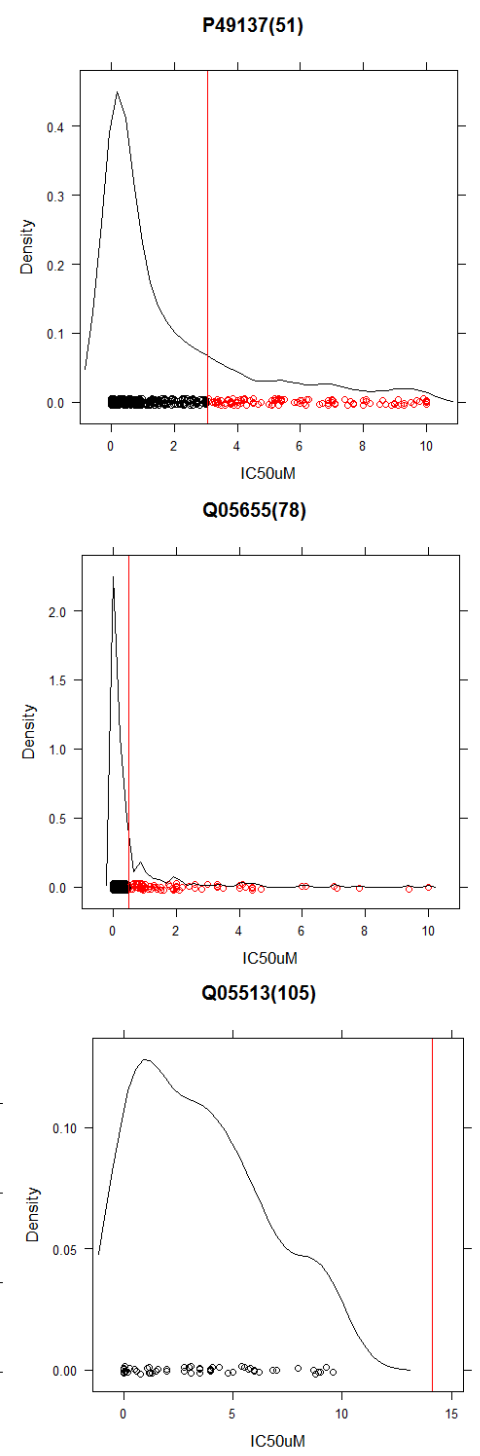

Q16539(3)

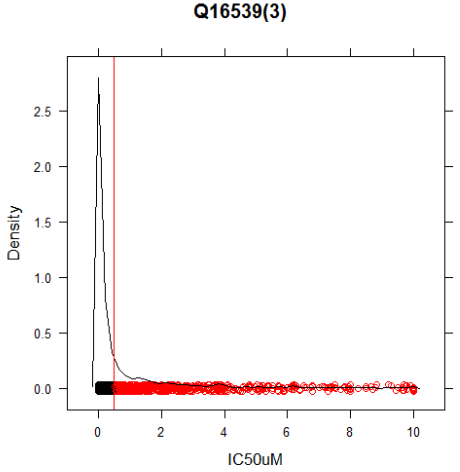

P36897(52)

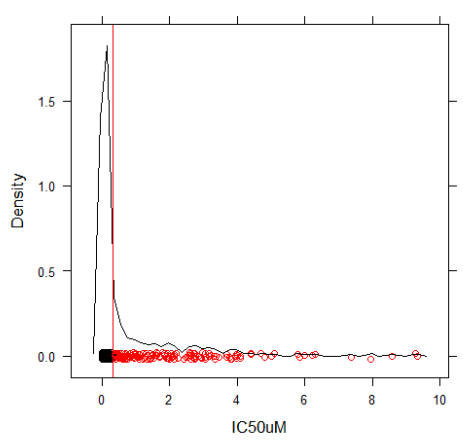

P53350(79)

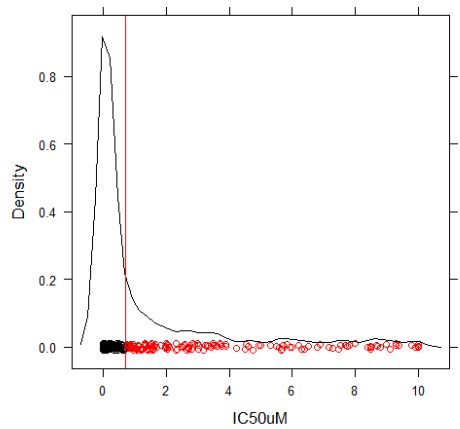

P34947(106)

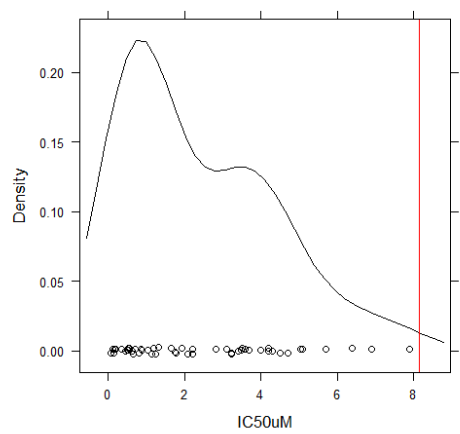

Figure S1. Density distribution plots of the activity values ( $\mathrm{IC}_{50}$ in $\mu \mathrm{M}$ ) and hot spot cutoff (red line) for the first three kinases (according to the ID number assigned in the paper) in cluster k1 (a), k2 (b), k3 (c) and k4 (d). The plot title contains the UniProt ID of the kinase and the ID number (in parenthesis) used in the paper. 


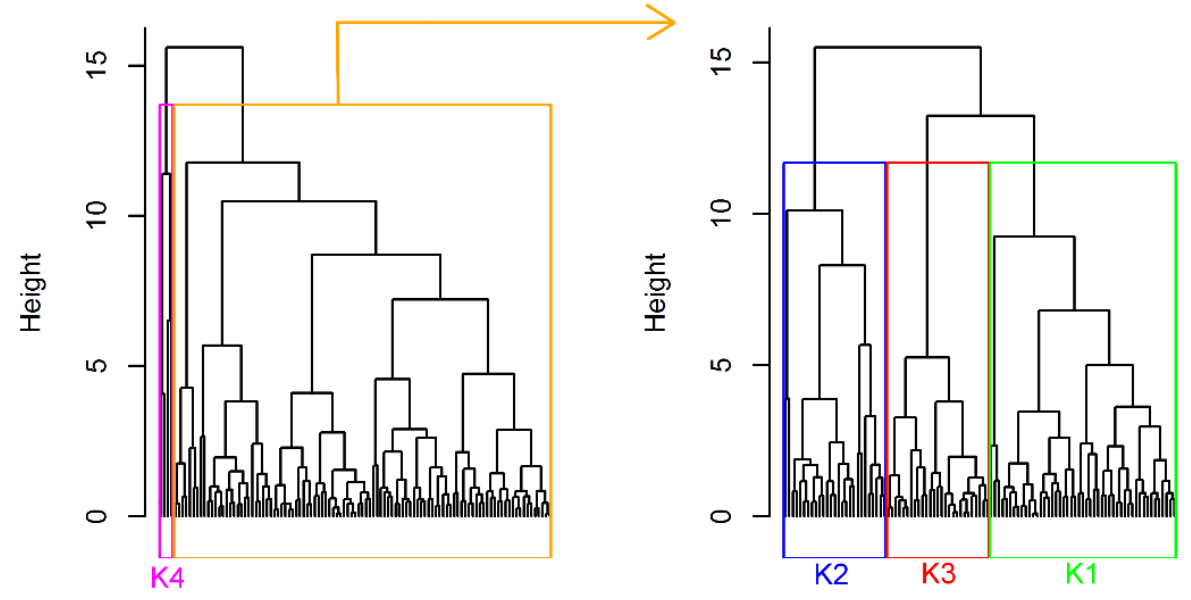

Figure S2. Dendrograms resulted from Ward hierarchical clustering of 107 kinase sets in a first run (left), resulting in k4 (magenta) and a larger cluster of 103 kinase sets. These were clustered separately into 3 more groups (right) k2 (blue), k3 (red) and k1 (green).

$$
\begin{gathered}
\text { Clusters - k1 } 4 \text { k2 }=\mathrm{k} 3+\mathrm{k} 4 \\
\text { Parameter - Acc_int } \cdots \cdots \text { Se_int ---- Sp_int }
\end{gathered}
$$

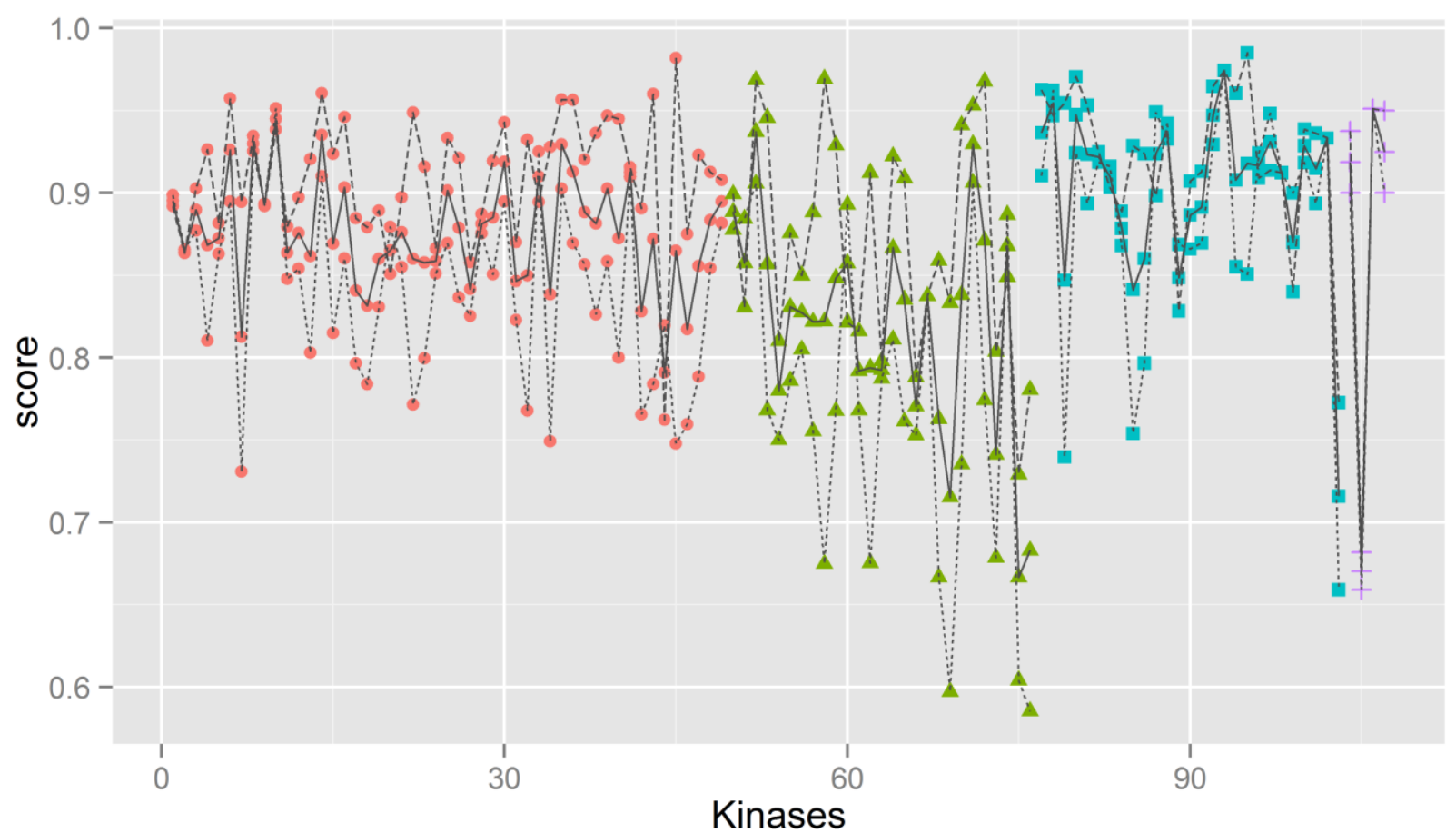

Figure S3. Accuracy (Acc), sensitivity (Se) and specificity (Sp) values of the internal (training sets) evaluation of the 107 kinase models. 


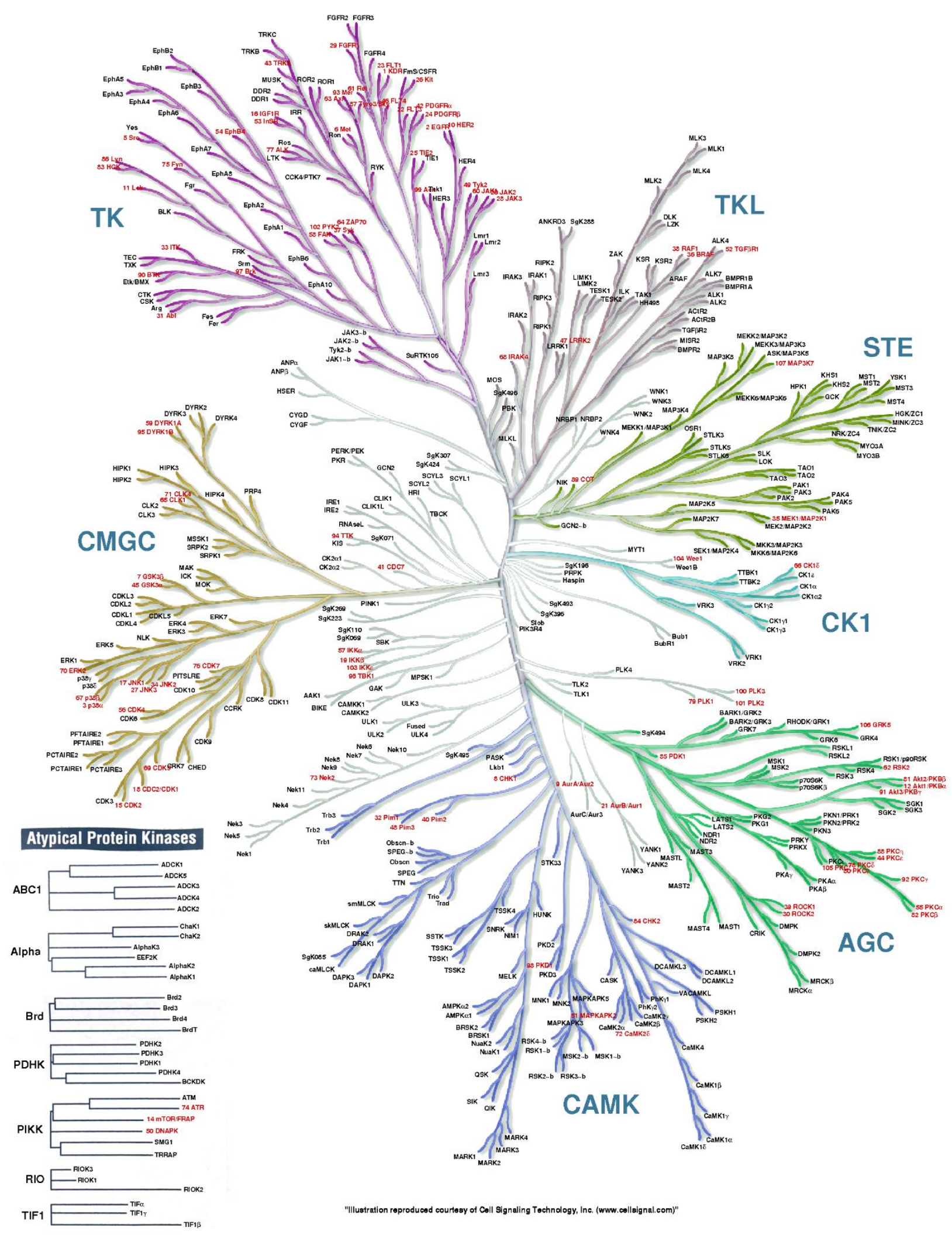

Figure S4. Phylogenetic tree of the human kinases (generated using Kinome Render ${ }^{13}$, 14) Target IDs and abbreviations of the kinases modeled in the current study are colored in red; two Atypical:PI3/PI4-kinase kinases, i.e., PIK3CA and PIK3CG could not be mapped and are not represented. Illustration reproduced courtesy of Cell Signaling Technology, Inc. (www.cellsignal.com). 

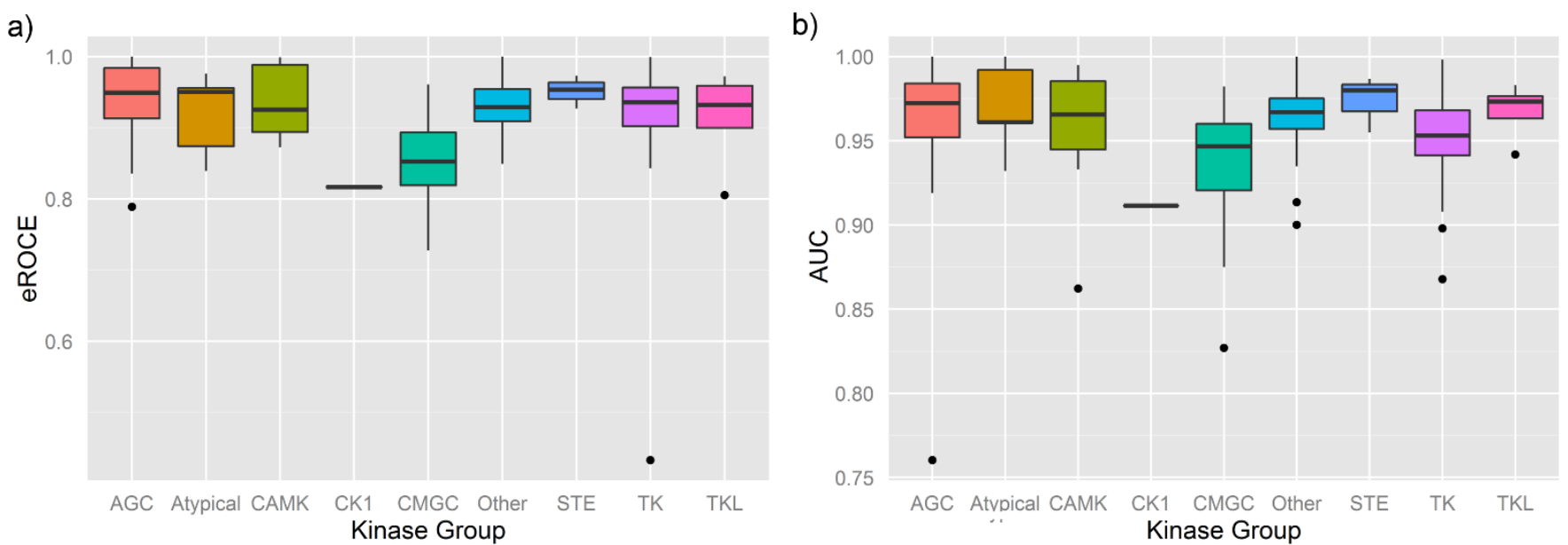

Figure S5. The distributions of (the kinase-set values) eROCE (a, performed on the VS test sets) and AUC (b, performed on the classification test sets) according to the nine kinase groups.

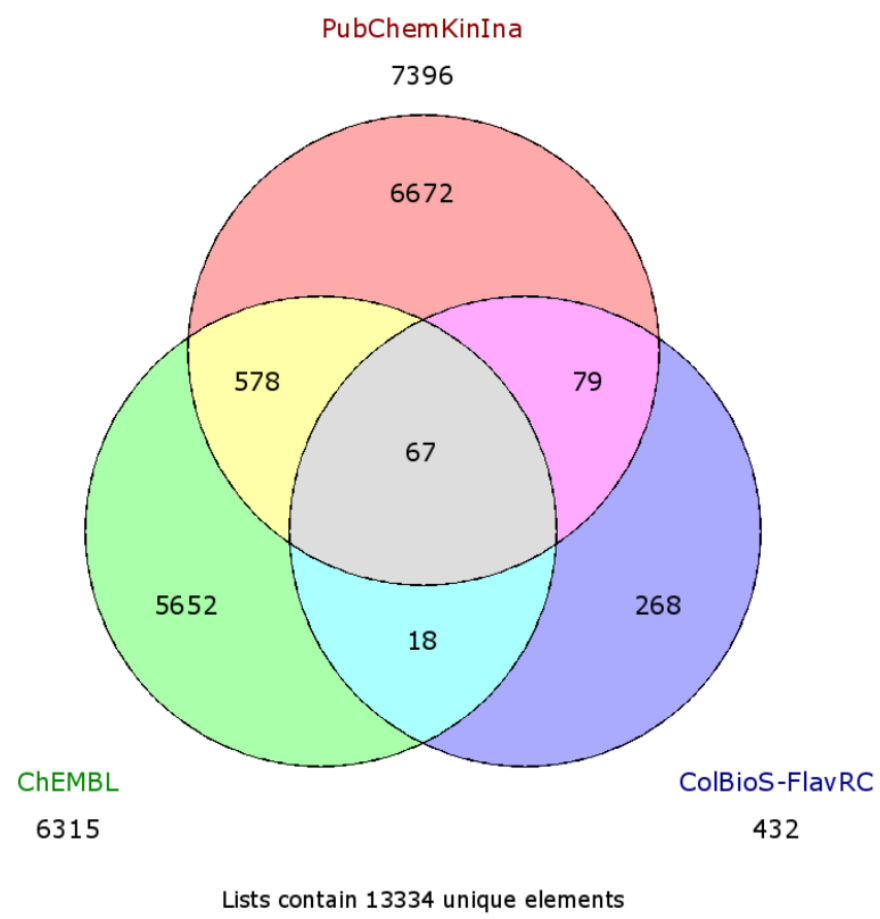

Figure S6. Venn plot (created using the online software from Microarray Center CRP-Sante) ${ }^{15}$ of the chemical scaffolds (BMFs) contained in the PubChemKinIna (only inactives), ChEMBL (only kinase inhibitors) and ColBioS-FlavRC ${ }^{16}$ datasets. 


\section{REFERENCES}

(1) UniProt, C., Reorganizing the Protein Space at the Universal Protein Resource (UniProt). Nucleic Acids Res. 2012, 40, D71-75.

(2) UniProtKB. http://www.uniprot.org/ (accessed Feb 4, 2015).

(3) Bemis, G. W.; Murcko, M. A. The Properties of Known Drugs. 1. Molecular Frameworks. J. Med. Chem. 1996, 39, 2887-2893.

(4) Manning, G.; Whyte, D. B.; Martinez, R.; Hunter, T.; Sudarsanam, S. The Protein Kinase Complement of the Human Genome. Science 2002, 298, 1912-1934

(5) ChemAxon JChem API package, version 6.1.0. 2013; ChemAxon (http//www.chemaxon.com).

(6) Strobl, C.; Hothorn, T.; Zeileis, A. Party on ! - A New, Conditional Variable Importance Measure for Random Forests Available in the party Package. The R Journal 2009, 1, 14-17.

(7) Strobl, C.; Malley, J.; Tutz, G. An Introduction to Recursive Partitioning: Rationale, Application, and Characteristics of Classification and Regression Trees, Bagging, and Random Forests. Psychol. Methods 2009, 14, 323-348.

(8) Strobl, C.; Boulesteix, A. L.; Zeileis, A.; Hothorn, T. Bias in Random Forest Variable Importance Measures: Illustrations, Sources and a Solution. BMC Bioinf. 2007, 8, 25.

(9) Hothorn, T.; Buhlmann, P.; Dudoit, S.; Molinaro, A.; van der Laan, M. J. Survival Ensembles. Biostatistics 2006, 7, 355-373.

(10) R Development Core Team. R: A Language and Environment for Statistical Computing, version 3.1.1; The R Foundation for Statistical Computing: Vienna, Austria, 2014; http://www.R-project.org/.

(11) Metz, J. T.; Johnson, E. F.; Soni, N. B.; Merta, P. J.; Kifle, L.; Hajduk, P. J. Navigating the Kinome. Nat. Chem. Biol. 2011, 7, 200-202 
(12) Anastassiadis, T.; Deacon, S. W.; Devarajan, K.; Ma, H.; Peterson, J. R. Comprehensive Assay of Kinase Catalytic Activity Reveals Features of Kinase Inhibitor Selectivity. Nat. Biotechnol. 2011, 29, 10391045.

(13) Chartier, M.; Chenard, T.; Barker, J.; Najmanovich, R. Kinome Render: a Stand-Alone and webAccessible Tool to Annotate the Human Protein Kinome Tree. Peer J. 2013, 1, e126.

(14) Najmanovich, R. Kinome Render [Online], http://bcb.med.usherbrooke.ca/kinomerender.php (accessed Sept 28, 2015).

(15) Microarray Center CRP-Sante [Online], http:/www.bioinformatics.lu/venn.php (accessed January 2016).

(16) Avram, S. I.; Pacureanu, L. M.; Bora, A.; Crisan, L.; Avram, S.; Kurunczi, L. ColBioS-FlavRC: A Collection of Bioselective Flavonoids and Related Compounds Filtered from High-Throughput Screening Outcomes. J. Chem. Inf. Model. 2014, 54, 2360-2370. 